Pacific Journal of Mathematics

SEVERAL DIMENSIONAL PROPERTIES OF THE SPECTRUM 


\title{
SEVERAL DIMENSIONAL PROPERTIES OF THE SPECTRUM OF A UNIFORM ALGEBRA
}

\author{
RICHARD F. BASENER
}

\begin{abstract}
The author has previously introduced a generalized Šilov boundary which seems useful in studying analytic structure of several dimensions in the spectrum of a uniform algebra $\mathfrak{A}$. Related generalizations of $\mathfrak{A}$-convexity, $\mathfrak{A}$-polyhedra, etc. are developed here. Several different but equivalent approaches to these various generalizations are described. The generalized boundaries discussed here are related to the " $q$ holomorphic functions" of the author, and to $\mathfrak{A}$-holomorphic convexity.
\end{abstract}

The generalized Šlov boundary was introduced by the author [2] to study multi-dimensional analytic structure in the spectrum of a uniform algebra. Related but more extensive applications of this boundary were developed by Sibony [13]. Kramm [10] has utilized this boundary to help obtain a characterization of Stein algebras. The definition of the Šilov boundary of order $q$ in [2] was motivated by consideration of $2($-varieties of codimension $q$ in the spectrum of 2 .

Here we show how extending $\mathfrak{A}$ by the conjugates of $q$ functions from $\mathfrak{A}$, decomposing the spectrum of $\mathfrak{A}$ into $q+1$ pieces, or generalizing the idea of an $\mathfrak{A}$-polyhedron all lead to the same circle of ideas as the $q$ th order boundary. We also relate this boundary to " $q$-holomorphic" functions. (In [3], [4] the author defined a function $f$ to be $q$-holomorphic if $\bar{\partial} f \wedge(\partial \bar{\partial} f)^{q}=0$, and developed some elementary properties of such functions.) Finally, we establish a connection between the first order boundary and the 2 -holomorphic convexity studied by Rickart [11].

We refer the reader to Stout's book, [14], for notation, terminology, and basic results concerning function algebras and uniform algebras.

1. Generalized boundaries and extension algebras, Let $A$ be a function algebra on the compact Hausdorff space $X$ (although the results of this section also apply if $X$ is locally compact). Let $\partial_{0} A$ denote the usual Šlov boundary for $A$. For a subset $S$ of $A$ let $\# S$ denote the cardinality of $S$ and let

$$
V(S)=\{x \in X \mid \forall f \in S, f(x)=0\} \text {. }
$$

If $K$ is a closed subset of $X$ define the restriction algebra 


$$
A \mid K=\left\{\left.f\right|_{K}: f \in A\right\}
$$

and let $A_{K}$ denote the uniform closure of $A \mid K$ in $C(K)$.

Definition. Let $q$ be a nonnegative integer. A subset $\Gamma$ of $X$ is a $q$ th order boundary for $A$ if given $S \subseteq A$ with $\# S \leqq q, V(S) \neq$ $\varnothing$, we have:

$$
\forall f \in A, \exists x \in \Gamma \cap V(S) \text { such that }|f(x)|=\max _{V(S)}|f| .
$$

We then define the $q$ th order Šilov boundary for $A$ by

$$
\partial_{q} A=\text { Closure }\left[\cup\left\{\partial_{0}[A \mid V(S)]: S \leqq A, \# S \leqq q\right\}\right] .
$$

Evidently $\partial_{q} A$ is the smallest closed $q$ th order boundary for $A$, and the two definitions for $\partial_{0} A$ are consistent.

Definition. If $\mathfrak{B}$ is a commutative Banach algebra with unit, let $M=M(\mathfrak{B})$ denote its spectrum and $\hat{B}$ its algebra of Gelfand transforms. Since $\hat{B}$ is a function algebra on $M$ we may define $\partial_{q} \mathfrak{B}=\partial_{q} \hat{B}$.

Now suppose that $A$ is a uniform algebra on the compact Hausdorff space $X$. We denote the corresponding commutative Banach algebra by $\mathfrak{A}$, and we identify $X$ with the corresponding subset of its spectrum $M$. Evidently $\partial_{q} A=\partial_{q} \mathfrak{A}$ if and only if $\partial_{q} \mathfrak{A} \subseteq X$. Of course $X$ contains the usual Šlov boundary of $A$, so this always holds for $q=0$, but it need not hold when $q>0$. (Let $\Delta=\{z \in C:|z| \leqq 1\}$. Take $X=\partial \Delta, A=P(X)$. Then $\partial_{q} A=X$ for all $q, \partial_{0} \mathfrak{A}=X$, but $\partial_{q} \mathfrak{A}=\Delta$ for $q>0$.) The generalized Šilov boundary used in [2], [10], and [13] is $\partial_{q} \mathfrak{A}$, but we shall sometimes find it more convenient here to use $\partial_{q} A$. For examples of $\partial_{q} \mathfrak{A}$, see [13], pp. 145-147.

Sibony apparently arrived at his definition of $\partial_{q} \mathfrak{A}$ by considering the behavior of plurisubharmonic functions. We include his definition here for completeness.

THeOREM 1 (Sibony, [13] Theorem 3). If $A$ is a uniform algebra on the compact Hausdorff space $X$, then $\partial_{q} \mathfrak{A}$ is the smallest compact subset of $M$ which satisfies the condition: whenever $f, g_{1}, \cdots, g_{q} \in A$ and $\operatorname{Re} f \leqq \sum_{j=1}^{q}\left|g_{j}\right|$ on $K$, then $\operatorname{Re} f \leqq \sum_{j=1}^{q}\left|g_{j}\right|$ on $M$.

When $\mathfrak{B}$ is a commutative Banach algebra with unit, $\partial_{q} \mathfrak{B}$ has an interpretation in terms of quotient algebras. To see this, recall that when $I$ is a closed ideal in $\mathfrak{B}$, the spectrum of $\mathfrak{B} / I$ is naturally identified with $V(\hat{I})=\{\varphi \in M,(\mathfrak{B}) \mid \forall f \in I, \widehat{f}(\varphi)=0\}$. Thus we obtain:

THEOREM 2. For a commutative Banach algebra $\mathfrak{B}$ with unit, 
$\partial_{q} \mathfrak{B}=$ Closure $\left[\cup\left\{\partial_{0}(\mathfrak{B} / I): I\right.\right.$ is an ideal of codimension at most $q$ in $\mathfrak{B}\}]$.

For the remainder of this section, we consider a function algebra $A$ on a compact Hausdorff space $X$, and show how the $q$ th order boundaries for $A$ are related to extensions of $A$ by conjugates of functions in $A$.

Notation. If $S \subseteq C(X)$, let $A(S)$ denote the function algebra on $X$ generated by $A$ and $S$; i.e.,

$$
A(S)=\left\{\sum_{|I| \leqq N} g_{I} f_{1}^{i_{1}} \cdots f_{r}^{i_{r}} \mid f_{1}, \cdots, f_{r} \in S, g_{I} \in A, 0 \leqq r, N<\infty\right\}
$$

where $I=\left(i_{1}, \cdots, i_{r}\right)$ and $|I|=i_{1}+\cdots+i_{r} ; i_{1}, \cdots, i_{r} \geqq 0$.

THeOREM 3. Let $\Gamma$ be a closed subset of $X$. Then $\Gamma$ is a $q$ th order boundary for $A$ if and only if for all $S \subseteq A$ with $\# S \leqq q, \Gamma$ is a boundary for $A(\bar{S})$.

Proof. First assume that $\Gamma$ is a $q$ th order boundary for $A$. Let $S=\left\{f_{1}, \cdots, f_{q}\right\} \subset A$, and let $F \in A(\bar{S})$, so that

$$
F=\sum_{I} g_{I} \bar{f}_{1}^{i_{1}} \cdots \bar{f}_{q}^{i_{q}}
$$

for some $g_{I} \in A$. Choose $y \in X$ with $|F(y)|=\max _{X}|F|$, and let

$$
\begin{aligned}
h_{j} & =f_{j}-f_{j}(y) \quad j=1, \cdots, q ; \\
T & =\left\{h_{1}, \cdots, h_{q}\right\} \leqq A ; \\
f & =\sum_{I} g_{I} \overline{f_{1}(y)^{i_{1}}} \cdots \overline{f_{q}(y)^{i_{q}}} \in A .
\end{aligned}
$$

Then $y \in V(T)$, so $V(T) \neq \varnothing$. Since $\Gamma$ is a $q$ th order boundary for $A, \max _{V(T)}|f|=\max _{V(T) \cap \Gamma}|f|$. But $y \in V(T)$ and $f=F$ on $V(T)$, whence $\max _{X}|F|=|F(y)|=\max _{\Gamma}|F|$ as desired.

Now suppose that for all $S \subseteq A$ with $\# S \leqq q, \Gamma$ is a boundary

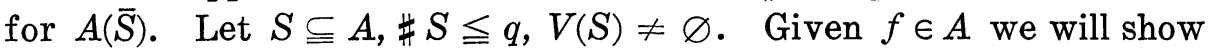
that $\max _{V(S)}|f|=\max _{V(S) \cap \Gamma}|f|$.

Let $S=\left\{f_{1}, \cdots, f_{q}\right\}$ and let $M=1+\max _{X} \sum_{j=1}^{q}\left|f_{j}\right|^{2}$. Set

$$
F=\frac{1}{M}\left(M-\sum_{j=1}^{q}\left|f_{j}\right|^{2}\right),
$$

and observe that $F=1$ on $V(S)$ while $0<F<1$ on $X \backslash V(S)$. For each $m \geqq 0$ we have $f F^{m} \in A(\bar{S})$, so that

$$
\max _{X}\left|f F^{m}\right|=\max _{\Gamma}\left|f F^{m}\right| \text {. }
$$


Since $F$ peaks on $V(S)$, it follows that

$$
\max _{V(S)}|f|=\max _{V(S) \cap \Gamma}|f| \text {. }
$$

2. Relationship with $q$-holomorphic functions. In [3], [4] we defined a function $f$ on $C^{n}$ to be $q$-holomorphic if $\bar{\partial} f \wedge[\partial \bar{\partial} f]^{q}=0$. The motivating example of such a function is one which is holomorphic in $(n-q)$ variables and arbitrary in the other $q$ variables. (Compare Example 4 and Theorem 1 in [3].) We showed that an $(n-1)$-holomorphic function on $C^{n}$ satisfies the maximum principle, and we related " $q$-holomorphic convexity" to $q$-pseudoconvexity (Theorems 2 and 3 of [3]). Hunt and Murray [9] have since related these $q$-holomorphic functions to the complex Monge-Ampere equations, obtaining results which extend Bremermann's work [6] on a generalized Dirichlet problem.

In order to develop some of the connections between the generalized Šilov boundary and the $q$-holomorphic functions, let us define

$$
\begin{aligned}
A(K)= & \{f \in C(K) \mid f \text { is holomorphic on } \operatorname{int} K\} \\
A^{q}(K)= & \left\{f \in C(K)|f|_{\text {int } K} \in C^{(2)}(\operatorname{int} K), f\right. \\
& \text { is } q \text {-holomorphic on int } K\}
\end{aligned}
$$

for $K$ an arbitrary compact subset of $C^{n}$. So, for example, $A^{0}(K)=$ $A(K)$ and $A^{n}(K)=\left\{f \in C(K)|f|_{\text {int } K} \in C^{(2)}\right.$ (int $\left.\left.K\right)\right\} . \quad A(K)$ is a uniform algebra but $A^{q}(K)$ is not even a linear space when $0<q<n$, although it does have some algebraic closure properties; for example, if $f \in A(K)$ and $g \in A^{q}(K)$, then $f+g, f g, g^{2} \in A^{q}(K)$ ([3], Proposition 4). We will still say that a subset $\Gamma$ of $K$ is a boundary for $A^{q}(K)$ if for all $f \in A^{q}(K), \max _{K}|f|$ is achieved on $\Gamma$. The maximum principle for $q$-holomorphic functions mentioned above shows that $\partial K$ is always a boundary for $A^{q}(K)$ when $0 \leqq q<n$, and certainly $K$ is the only boundary for $A^{q}(K)$ when $q \geqq n$. Similarly, it is clear that $\partial K$ is a $q$ th order boundary for $A(K)$ when $0 \leqq q<n$, and that the only $q$ th order boundary for $A(K)$ when $q \geqq n$ is $K$. One reason for this similarity is given by the following result.

THEOREM 4. Let $\Gamma$ be a closed subset of the compact set $K \subseteq C^{n}$. If $\Gamma$ is a boundary for $A^{q}(K)$, then $\Gamma$ is a qth order boundary for $A(K)$.

Proof. Let $S \subseteq A, \# S \leqq q$. It is easy to verify that $A(K)(\bar{S}) \leqq$ $A^{q}(K)$. Since $\Gamma$ is a boundary for $A^{q}(K)$, it is a boundary for $A(K)(\bar{S})$. By Theorem 3, $\Gamma$ is a $q$ th order boundary for $A(K)$.

Now suppose that $\Omega$ is a bounded open subset of $C^{n}$ with $C^{2}$ 
boundary. Recall that $\Omega$ is (strictly) $q$-pseudoconvex at a point $x \in$ $\partial \Omega$ if the Levi form in the complex tangent space to $\Omega$ at $x$ of a defining function for $\Omega$ has at least $n-1-q$ nonnegative (positive) eigenvalues. Let

$$
F_{q, \Omega}=\text { Closure }\{x \in \partial \Omega \mid \Omega \text { is strictly } q \text {-pseudoconvex at } x\} .
$$

THEOREM 5. Let $\Omega$ be a bounded open subset of $C^{n}$ with $C^{2}$ boundary. Then $F_{q, \Omega}$ is a boundary for $A^{q}(\bar{\Omega})$.

Proof. For $q=0$, see Epe [7] (or [5] or [8]). The same argument used in, say, [5] can be applied when $q>0$. We outline a proof, based on this argument, for the case $0<q<n$.

Let $f \in A^{q}(\bar{\Omega})$; we will show that $\max _{\bar{\Omega}}|f|=\max _{F_{q}, \Omega}|f|$. By the closure properties of $A^{q}(\bar{\Omega})$ mentioned above, we know that $A(\bar{\Omega})(\{f\}) \cong A^{q}(\bar{\Omega})$. Let $B$ denote the uniform closure of $A(\bar{\Omega})(\{f\})$, so that $B$ is a uniform algebra on $\bar{\Omega}$. We will show that $F_{q, \Omega}$ contains $\partial_{0} B$, which will complete the proof. For this it suffices to show that any peak point $x \in \partial \Omega$ for $B$ is a limit of strictly $q$-pseudoconvex boundary points of $\Omega$. Now given any small neighborhood $U$ of such an $x$, there is a $g \in A(\bar{\Omega})(\{f\})$ for which $\operatorname{Re} g$ achieves its maximum value, say 1 , only in $U$. Since $\operatorname{Re} g$ is $q$-plurisubharmonic on $\Omega$ (Theorem 3.3 of [9]), $\varphi(z)=-1+\varepsilon \sum_{j=1}^{n}\left|z_{j}\right|^{2}+\operatorname{Re} g(z)$ is strictly $q$-plurisubharmonic on $\Omega$ for any positive $\varepsilon$. If we choose $\varepsilon$ to be a small positive number, and $c$ to be a small negative number for which $W=\{z \in \Omega \mid \varphi(z)=c\}$ is smooth, and if we then translate the hypersurface $W$ in the outward normal direction to $\Omega$ at $x$ until $W$ is externally tangent to $\Omega$, any point of tangency of $W$ provides a strictly $q$-pseudoconvex boundary point of $\Omega$ near $x$.

Note. There does not seem to be a simple way to apply the above argument directly to the original function $f \in A^{q}(\bar{\Omega})$, as the set $\left\{z \in \partial \Omega \mid \operatorname{Re} f(z)=\max _{\bar{\Omega}} \operatorname{Re} f\right\}$ may extend over a large portion of $\partial \Omega$. Then we cannot simply translate a level hypersurface to make it externally tangent.

Putting Theorems 4 and 5 together, we see that $F_{q, \Omega}$ always contains $\partial_{q} A(\bar{\Omega})$. In fact, Sibony has shown that $\partial_{q} A(\bar{\Omega})=F_{q, \Omega}$ when $\Omega$ is a $C^{\infty}$ pseudoconvex domain which is an " $S_{\delta}$ ". ([13], Proposition 4.) In this case $\bar{\Omega}$ is the spectrum of the corresponding Banach algebra $\mathfrak{A}(\bar{\Omega})$, so we also have $\partial_{q} \mathfrak{R}(\bar{\Omega})=F_{q, \Omega}$. Furthermore, it is easy to see that $F_{q, \Omega}$ is the smallest closed boundary for $A^{q}(\Omega)$ in this case. For an arbitrary bounded $\Omega$ with $C^{2}$ boundary it would seem to be a difficult question to determine whether a given strictly $q$ pseudoconvex boundary point $x$ of $\Omega$ must be included in every closed 
boundary for $A^{q}(\bar{\Omega})$ or in $\partial_{q} A(\bar{\Omega})$, as these involve global existence questions; but it is not hard to see that for any such $x$ there is a closed ball $B$ centered at $x$ for which $x \in \partial_{q} A(\bar{\Omega} \cap B)$ and for which $x$ is any closed boundary for $A^{q}(\bar{\Omega} \cap B)$. (See the proof of Theorem 3 in [3] for the construction of an appropriate peaking function.)

3. Generalizations of $\mathscr{A}$-convexity. Throughout this section let $A$ be a uniform algebra on the compact Hausdorff space $X$. As in section one, $\mathfrak{A}$ denotes the corresponding Banach algebra and $M$ denotes its spectrum; we will also regard $\mathfrak{A}$ as a uniform algebra on $M . K, K_{j}$, etc. will always denote closed subsets of $M$. We recall briefly some facts about $\mathfrak{A}$-convexity.

The $\mathfrak{A}$-convex hull of $K$ is defined by

$$
h(K)=\left\{x \in M|\forall f \in \mathfrak{A},| f(x)\left|\leqq \max _{K}\right| f \mid\right\},
$$

and the rational $\mathfrak{A}$-convex hull of $K$ is

$$
r h(K)=\{x \in M \mid \forall f \in \mathfrak{A}, f(x) \in f(K)\} .
$$

$K$ is a boundary for $\mathfrak{A}$ if and only if $h(K)=M$. One says that a set $K$ is $\mathfrak{A}$-convex if and only if $h(K)=K$. The simplest $\mathfrak{A}$-convex sets are the $\mathfrak{A}$-polyhedra. If $D=\{|z| \leqq 1\}$ and if $F_{1}, \cdots, F_{r} \in \mathfrak{A}$, the corresponding $\mathfrak{A}$-polyhedron is

$$
\pi\left(F_{1}, \cdots, F_{r}\right)=\left\{x \in M \mid F_{j}(x) \in D, j=1, \cdots, r\right\} .
$$

$h(K)=\bigcap\{\pi: \pi \supseteqq K, \pi$ is an $\mathscr{A}$-polyhedron $\}$.

There is an obvious generalization of $h(K)$ parallel to the generalized Šilov boundary.

DEFINITION.

$$
\begin{aligned}
h_{q}(K)= & \{x \in M \mid \forall S \leqq \mathfrak{A} \text {, if } \# S \leqq q \text { and } x \in V(S), \\
& \text { then } \left.\forall f \in \mathfrak{A},|f(x)| \leqq \max _{V(S) \cap K}|f|\right\} .
\end{aligned}
$$

(Here $V(S)=\{x \in M \mid \forall f \in S, f(x)=0\}$.) Evidently $K$ is a $q$ th order boundary for the algebra $\mathfrak{A}$ on $M$ if and only if $h_{q}(K)=M$.

A similar generalization of $\mathfrak{A}$-polyhedron is also possible, and in fact one was made by Rothstein [12] in studying Hartogs' theorems for analytic varieties. Our definition is based on his. Let

$$
D^{n}=\left\{z \in C^{n} \mid z=\left(z_{1}, \cdots, z_{n}\right) \text {, and for some } j,\left|z_{j}\right| \leqq 1\right\},
$$

and let $\mathfrak{A}^{n}=\left\{F=\left(f_{1}, \cdots, f_{n}\right) \mid f_{1}, \cdots, f_{n} \in \mathfrak{A}\right\}$. 
Definition. If $F_{1}, \cdots, F_{r} \in \mathfrak{Q}^{q+1}$, the corresponding $q$-polyhedron is

$$
\pi\left(F_{1}, \cdots, F_{r}\right)=\left\{x \in M \mid F_{j}(x) \in D^{q+1}, j=1, \cdots, r\right\} .
$$

Note for future reference that the $q$-polyhedra are precisely the subsets of $M$ which are finite intersections of unions of $q+1 \mathfrak{A}-$ polyhedra; for example, if $F=\left(f_{1}, \cdots, f_{q+1}\right)$, then $\pi(F)=\bigcup_{j=1}^{q+1} \pi\left(f_{j}\right)$.

The $q$-polyhedra are related to $h_{q}(K)$ in the same way that $\mathfrak{A}$ polyhedra are related to $h(K)$. In proving this we will make use of some alternative descriptions of $h_{q}(K)$, two of which are based on decomposing $K$ into $q+1$ pieces and examining their hulls. We need a preliminary lemma which describes this kind of decomposition in $\boldsymbol{C}^{q}$.

Lemma. If $B^{n}=\left\{z \in C^{n}|| z \mid \leqq 1\right\}$, then there are compact polynomially convex sets $L_{0}, L_{1}, \cdots, L_{n} \subseteq B^{n}$ such that:

(i ) $B^{n}=\bigcup_{j=0}^{n} L_{j}$ and

(ii) 0 is a peak point for $P\left(L_{j}\right), j=0, \cdots, n$.

Such a decomposition is not possible with fewer than $n+1$ subsets of $B^{n}$. (Here $|z|=\left(\Sigma\left|z_{j}\right|^{2}\right)^{1 / 2}$.)

Proof. Let

$$
\begin{aligned}
M_{j}= & \left\{z \in C^{n} \mid \text { for each nonzero coordinate } z_{i} \text { of } z,\right. \\
& \left.\frac{2 \pi j}{n+1} \leqq \arg z_{i} \leqq \frac{2 \pi(n+j)}{n+1}\right\}, \quad k=0, \cdots, n .
\end{aligned}
$$

Each $M_{j}$ is a product of one dimensional sectors about the origin, and $\mathrm{U}_{s=0}^{n} M_{j}=C^{n}$. It follows that

$$
L_{j}=M_{j} \cap B^{n}, \quad j=0, \cdots, n
$$

yields the desired decomposition. That $n+1$ pieces are needed will follow from the next result applied to $P\left(B^{n}\right)$, since $\partial_{n-1}\left(P\left(B^{n}\right)\right)=\partial B^{n}$. As a final preliminary, suppose $S \subseteq \mathfrak{A}$ and define

$$
h_{S}(K)=\left\{x \in M|\forall f \in \mathfrak{A}(\bar{S}),| f(x)\left|\leqq \max _{K}\right| f \mid\right\} .
$$

Of course this is just the $\mathfrak{B}$-convex hull of $K$, where $B$ is the uniform algebra generated by $A$ and $\{\bar{f}: f \in S\}$.

THEOREM 6. For any closed subset $K$ of $M$, the following sets are equal: 


$$
\begin{aligned}
H_{1}= & h_{q}(K) ; \\
H_{2}= & \bigcap\left\{h_{S}(K) \mid S \subseteq \mathfrak{A}, \# S \leqq q\right\} ; \\
H_{3}= & \bigcap\{\pi \mid \pi \text { is a q-polyhedron containing } K\} ; \\
H_{4}= & \left\{x \in M \mid \text { for any decomposition } K=\bigcup_{j=0}^{q} K_{j}, x \in \bigcup_{j=0}^{q} h\left(K_{j}\right)\right\} ; \\
H_{5}= & \left\{x \in M \mid \text { if } K_{1}, \cdots, K_{q} \leqq K \text { and } x \notin \bigcup_{j=1}^{q} r h\left(K_{j}\right),\right. \text { then there } \\
& \text { is a compact set } \left.L \subseteq K \backslash \bigcup_{j=1}^{q} K_{j} \text { with } x \in h(L)\right\} .
\end{aligned}
$$

Proof. $H_{1}=H_{2}$ : This follows readily from the definitions by considering $\left.A\right|_{H_{1}}$ and $\left.A\right|_{H_{2}}$ together with Theorem 3.

$H_{1} \leqq H_{5}$ : Let $x \in h_{q}(K)$, let $K_{1}, \cdots, K_{q} \leqq K$, and assume $x \notin$ $\bigcup_{j=1}^{q} r h\left(K_{j}\right)$. We will exhibit a compact set $L \subseteq K, L$ disjoint from $K_{1}, \cdots, K_{q}$, with $x \in h(L)$.

For $j=1, \cdots, q$ choose $f_{j} \in \mathfrak{A}$ with $0=f_{j}(x) \notin f_{j}\left(K_{j}\right)$. Let $S=$ $\left\{f_{1}, \cdots, f_{q}\right\}$. Then $x \in V(S) \cap h_{q}(K)$, so $\forall f \in \mathfrak{A},|f(x)| \leqq \max _{V(S) \cap K}|f|$. $L=V(S) \cap K$ has the desired properties.

$H_{5} \subseteq H_{4}$ : This is obvious.

$H_{4} \leqq H_{1}$ : Let $x \in H_{4}$, let $S=\left\{f_{1}, \cdots, f_{q}\right\} \subseteq \mathfrak{A}$, and assume $x \in$ $V(S)$. We will show that $x \in h(V(S) \cap K)$. Assume $\Sigma\left|f_{i}\right|^{2} \leqq 1$.

By the above lemma there are compact polynomially convex sets $L_{0}, \cdots, L_{q} \subseteq B^{q}$ with $B^{q}=\bigcup_{j=0}^{q} L_{j}$ and 0 a peak point for $P\left(L_{j}\right), j=0, \cdots, q$. Let

$$
K_{j}=\left\{x \in K \mid\left(f_{1}(x), \cdots, f_{q}(x)\right) \in L_{j}\right\}, \quad j=0, \cdots, q .
$$

Since $x \in H_{4}$, there is a $j$ such that $x \in h\left(K_{j}\right)$. Let $\psi$ be a function in $P\left(L_{j}\right)$ which peaks at 0 , and let $\Psi=\psi\left(f_{1}, \cdots, f_{q}\right)$. Then $\Psi \in \mathfrak{A}_{K_{j}}$, the uniform closure of the restriction algebra $\left.\mathfrak{A}\right|_{K_{j}}$. From the facts that $x \in V(S) \cap h\left(K_{j}\right)$ and that $\Psi$ peaks on $V(S) \cap h\left(K_{j}\right)$, it follows that any representing measure for $x$ on $K_{j}$ is supported on $V(S) \cap K_{j}$. Thus $x \in h\left(V(S) \cap K_{j}\right) \subseteq h(V(S) \cap K)$ as desired.

$H_{4} \subseteq H_{3}$ : Suppose $x \notin H_{3}$. Let $\pi$ be a $q$-polyhedron for which $K \subseteq \pi$ but $x \notin \pi$. As noted above, $\pi$ can be written in the form $\pi=\bigcap_{i} \bigcup_{j=0}^{q} \pi_{i j}$, where the $\pi_{i j}$ are $\mathfrak{A}$-polyhedra. Then for some $i$ we have $x \notin \bigcup_{j=0}^{q} \pi_{i j}$. Let $K_{j}=K \cap \pi_{i j}, j=0, \cdots, q$. Evidently $K=$ $\bigcup_{j=0}^{q} K_{j}$ and $x \notin \bigcup_{j=0}^{q} \pi_{i j} \supseteq \bigcup_{j=0}^{q} h\left(K_{j}\right)$, so $x \notin H_{4}$.

$H_{3} \cong H_{4}:$ Suppose $x \notin H_{4}$. Then there are $K_{0}, \cdots, K_{q}$ with $K=$ 
$K_{j}, x \notin \bigcup U h\left(K_{j}\right)$. Choose $f_{j} \in \mathfrak{A}$ with $\left|f_{j}(x)\right|>1 \geqq \max _{K_{j}}\left|f_{j}\right|, j=$ $0, \cdots, q$. Let $F=\left(f_{0}, \cdots, f_{q}\right)$. Then $x \notin \pi(F) \supseteqq K$, so $x \notin H_{3}$.

CoROLLARY. $\partial_{q} \mathfrak{A}$ is the smallest compact subset $K$ of $M$ having the property: for every decomposition of $K$ into $q+1$ compact subsets, $K=\bigcup_{j=0}^{q} K_{j}$, one has $\bigcup_{j=0}^{q} h\left(K_{j}\right)=M$.

4. 2A-holomorphic convexity and the first order boundary. Again let $A$ denote a uniform algebra on $X$, with $M, \mathfrak{A}$ as in section three. Since the higher order boundaries reflect higher dimentional structure in $M$, and since holomorphic convexity first becomes interesting in $C^{2}$, it is reasonable to expect some connection between the first order boundary and uniform algebra generalizations of holomorphic convexity. An appropriate notion of $\mathfrak{Q}$-holomorphic convexity was studied by Rickart [11], which we now recall.

Definition. Let $U$ be an open subset of $M$ and let $\mathscr{O}(U)$ denote the locally $\mathscr{Q}$-holomorphic functions on $U$, i.e., $\mathcal{O}(U)=\{f \in C(U) \mid \forall x \in$ $U \exists$ a compact neighborhood $N$ of $x$ such that $\left.\left.f\right|_{N} \in \mathfrak{A}_{N}\right\}$. For a compact set $K \subseteq U$, set

$$
\hat{K}=\left\{x \in U|\forall f \in \mathcal{O}(U),| f(x)\left|\leqq \max _{K}\right| f \mid\right\} .
$$

Then $U$ is called $\mathscr{2}$-holomorphically convex if for all compact sets $K \subseteq U, \hat{K}$ is compact.

THEOREM 7. There are no proper $\mathfrak{X}$-holomorphically convex open subsets of $M$ containing $\partial_{1} \mathfrak{A}$.

Proof. Let $U$ be an open subset of $M$ contaiging $\partial_{1} \mathfrak{A}$. Assume $K=M \backslash U \neq \varnothing$. We will show that $U$ is not $\mathfrak{A}$-holomorphically convex by showing that $\left(\partial_{1} \mathfrak{A}\right)^{\wedge}$ is not compact.

Let $x$ be a peak point for $\mathfrak{A}_{K}$. Then $x \in K$, and the local maximum modulus principle implies that $x \in \partial[h(K)]$. Choose $x_{\alpha} \in M \backslash h(K)$ with $x_{\alpha} \rightarrow x$, and for each $\alpha$ choose $f_{\alpha} \in \mathfrak{A}$ with $f_{\alpha}\left(x_{\alpha}\right)=1>\max _{K}\left|f_{\alpha}\right|$. Fix $\alpha$ and take $S=\left\{f_{\alpha}-1\right\}$. Then $x_{\alpha} \in V(S) \leqq U$, and $\partial_{0}\left[\mathfrak{A}_{V(S)}\right] \leqq \partial_{1} \mathfrak{A}$, whence (using, say, Corollary 28.9 in [14]) $x_{\alpha} \in\left(\partial_{1} \mathfrak{A}\right)^{\wedge}$. Thus $\left(\partial_{1} \mathfrak{A}\right)^{\wedge}$ is not compact.

Let us say that a compact set $K \cong M$ is "large" when the only 2-holomorphically convex open set containing $K$ is $M$, so that the content of Theorem 7 is that $\partial_{1} \mathfrak{A}$ is always large. Clearly any large set must contain $\partial_{0} \mathfrak{A}$, so that when $\partial_{0} \mathfrak{A}=\partial_{1} \mathfrak{A}$, this is the smallest large subset of $M$. (This happens, e.g., for $A=P\left(B^{n}\right), n \geqq 2$.) When $\partial_{0} \mathfrak{A} \neq \partial_{1} \mathfrak{A}$, it may happen that there is a smallest large set $K$ with 
either $K=\partial_{0} \mathfrak{A}$ or $K=\partial_{1} \mathfrak{A}$ or $\partial_{0} \mathfrak{A} \subsetneq K \sqsubseteq \partial_{1} \mathfrak{A}$; or there may be no smallest large set. For example, if $A=P\left(\Delta^{1}\right)$ (where $\Delta^{n}=\left\{z \in C^{n}|| z_{j} \mid \leqq 1\right\}$ ), then $\partial_{0} \mathfrak{A}=\partial \Delta^{1}$, but $\partial_{1} \mathfrak{A}=\Delta^{1}$ is the smallest large set. If $A=R(X)$ where $X$ is one of the compact subsets of $\partial \Delta^{2}$ in [1] or [15], $\partial_{0} \mathfrak{A}=X$ is the smallest large set while $\partial_{1} \mathfrak{A}=h_{r}(X) \neq X$. Finally, consider $A=P\left(\Delta^{2}\right), K_{1}=\partial_{1} \mathfrak{A}=\partial \Delta^{2}, K_{2}=\left\{(z, w) \in \Delta^{2}|| z \mid=1\right.$ or $\left.|z|=|w|\right\}, K_{3}=$ $\left\{(z, w) \in \Delta^{2}|| w \mid=1\right.$ or $\left.|w|=|z|\right\}$. Then $K_{1}, K_{2}, K_{3}$ are all large, but $K_{1} \cap K_{2} \cap K_{3}=\partial_{0} \mathfrak{A}$ is not large.

\section{REFERENCES}

1. R. Basener, On rationally convex hulls, Trans. Amer. Math. Soc., 182 (1973), 353381.

2. - A generalized Šilov boundary and analytic structure, Proc. Amer. Math. Soc., 47 (1975), 98-104.

3. - Nonlinear Cauchy-Riemann equations and q-pseudoconvexity, Duke Math. J., 43 (1976), 203-213.

4. - Nonlinear Cauchy-Riemann equations and q-convexity, Proc. Sympos. Pure Math., Vol. 30, Part 1, Amer. Math. Soc., Providence, R. I., (1977), 3-5.

5. — Peak points, barriers, and pseudoconvex boundary points, Proc. Amer. Math. Soc., 65 (1977), 89-92.

6. H. J. Bremermann, On a generalized Dirichlet problem for plurisubharmonic functions and pseudoconvex domains, characterization of Šilov boundaries, Trans. Amer. Math. Soc., 91 (1959), 246-276.

7. R. Epe, Charakterisierung des Schilovrandes von Holomorphiegebieten Schr. Math. Inst. Univ. Munster, 25, 68 (1963).

8. M. Hakim and N. Sibony, Frontière de Šilov et spectre de $A(\bar{D})$ pour des domaines faiblement pseudoconvexes, C. R. Acad. Sc. Paris, 281 (1975), 959-962.

9. L. R. Hunt and J. Murray, A generalized Dirichlet problem for q-pseudoconvex domains, preprint.

10. B. Kramm, Eine funktionalanalytische charakterisierung der Steinschen algebren, preprint.

11. C. Rickart, Holomorphic convexity for general function algebras, Canad. J. Math., 20 (1968), 272-290.

12. Rothstein, Zur Theorie der analytischen Mannigfaltigkeiten in Raume von $n$ komplexen Veränderlichen, Math. Ann., 129 (1955), 96-138.

13. N. Sibony, Multi-dimensional analytic structure in the spectrum of a uniform algebra, Spaces of Analytic Functions (Kristiansand, Norway 1975), Lecture Notes in Math., no. 512, Springer-Verlag, Berlin and New York, (1976), 139-165.

14. E. L. Stout, The Theory of Uniform Algebras, Bogden and Quigley, Tarrytownon-Hudson, 1971.

15. J. Wermer, On an example of Stolzenberg, Sympos. Several Complex Variables (Park City, Utah, 1970), Lecture Notes in Math., no. 184, Springer-Verlag, Berlin and New York, (1971), 79-84.

Received June 24, 1977. This research was supported in part by NSF Grant MCS 76-04661. 


\section{PACIFIC JOURNAL OF MATHEMATICS}

\section{EDITORS}

RICHARD ARENS (Managing Editor)

University of California

Los Angeles, CA 90024

Charles W. Curtis

University of Oregon

Eugene, OR 97403

C. C. Moore

University of California

Berkeley, CA 94720

\section{J. DugundJI}

Department of Mathematics

University of Southern California

Los Angeles, CA 90007

R. FInN and J. Milgram

Stanford University

Stanford, CA 94305

\section{ASSOCIATE EDITORS}
E. F. BECKENBACH
B. H. NeumanN
F. WOLF
K. YoSHIDA

\section{SUPPORTING INSTITUTIONS}

\author{
UNIVERSITY OF BRITISH COLUMBIA \\ CALIFORNIA INSTITUTE OF TECHNOLOGY \\ UNIVERSITY OF CALIFORNIA \\ MONTANA STATE UNIVERSITY \\ UNIVERSITY OF NEVADA, RENO \\ NEW MEXICO STATE UNIVERSITY \\ OREGON STATE UNIVERSITY \\ UNIVERSITY OF OREGON
}

\author{
UNIVERSITY OF SOUTHERN CALIFORNIA \\ STANFORD UNIVERSITY \\ UNIVERSITY OF HAWAII \\ UNIVERSITY OF TOKYO \\ UNIVERSITY OF UTAH \\ WASHINGTON STATE UNIVERSITY \\ UNIVERSITY OF WASHINGTON
}

The Supporting Institutions listed above contribute to the cost of publication of this Journal, but they are not owners or publishers and have no responsibility for its content or policies.

Mathematical papers intended for publication in the Pacific Journal of Mathematics should be in typed form or offset-reproduced, (not dittoed), double spaced with large margins. Please do not use built up fractions in the text of the manuscript. However, you may use them in the displayed equations. Underline Greek letters in red, German in green, and script in blue. The first paragraph or two must be capable of being used separately as a synopsis of the entire paper. Items of the bibliography should not be cited there unless absolutely necessary, in which case they must be identified by author and journal, rather than by item number. Manuscripts, in triplicate, may be sent to any one of the editors. Please classify according to the scheme of Math. Reviews, Index to Vol. 39. All other communications should be addressed to the managing editor, or Elaine Barth, University of California, Los Angeles, California, 90024.

50 reprints to each author are provided free for each article, only if page charges have been substantially paid. Additional copies may be obtained at cost in multiples of 50 .

The Pacific Journal of Mathematics is issued monthly as of January 1966. Regular subscription rate: $\$ 72.00$ a year (6 Vols., 12 issues). Special rate: $\$ 36.00$ a year to individual members of supporting institutions.

Subscriptions, orders for numbers issued in the last three calendar years, and changes of address should be sent to Pacific Journal of Mathematics, 103 Highland Boulevard, Berkeley, California, 94708. Older back numbers obtainable from Kraus Periodicals Co., Route 100, Millwood, NY 10546.

PUBLISHED BY PACIFIC JOURNAL OF MATHEMATICS, A NON-PROFIT CORPORATION

Printed at Kokusai Bunken Insatsusha (International Academic Printing Co., Ltd.). 8-8, 3-chome, Takadanobaba, Shinjuku-ku, Tokyo 160, Japan.

Copyright (C) 1978 by Pacific Journal of Mathematics

Manufactured and first issued in Japan 


\section{Pacific Journal of Mathematics

Vol. 74, No. $2 \quad$ June, 1978

Aharon Atzmon, Spectral synthesis in some spaces of bounded continuous

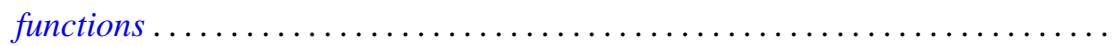

Karl Egil Aubert and Isidor Fleischer, Tensor products of ideal systems and their modules.............................................

Richard F. Basener, Several dimensional properties of the spectrum of a uniform

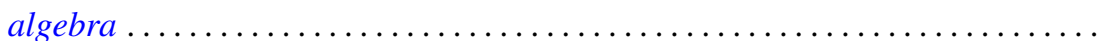

R. H. Bing and Michael Peter Starbird, Super triangulations ............. 307

Andrew Carson, Coherent polynomial rings over regular rings of finite index .....

Robert M. DeVos and Frederick W. Hartmann, Sequences of bounded summability domains .................................................

George Grätzer and R. Padmanabhan, Symmetric difference in abelian groups ....

Robert L. Griess, Jr., A remark about groups of characteristic 2-type and

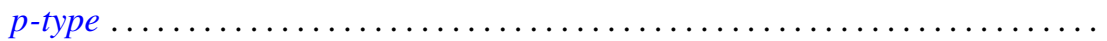

Emil Grosswald and F. J. Schnitzer, A class of modified $\zeta$ and L-functions........

Jutta Hausen and Johnny Albert Johnson, Ideals and radicals of some

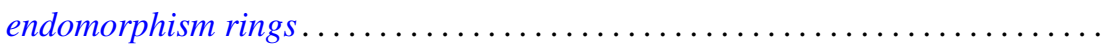

Jean Ann Larson, A solution for scattered order types of a problem of Hagendorf ............................................

Peter A. McCoy, Extremal properties of real biaxially symmetric potentials in $E^{2(\alpha+\beta+2)}$

Héctor Alfredo Merklen, Hereditary crossed product orders .

Hal G. Moore and Adil Mohamed Yaqub, Equational definability of addition in certain rings...

Robert Laurens Moore, Reductivity in $C^{*}$-algebras and essentially reductive operators. . .

Joseph Alvin Neisendorfer, Lie algebras, coalgebras and rational homotopy theory for nilpotent spaces...

William Raymond Nico, Bounded monoids

Richard Paul Osborne, Simplifying spines of 3-manifolds ...

Richard Paul Osborne, The simplest closed 3-manifolds. With an appendix by Osborne and J. Yelle.

Clayton Collier Sherman, The $K$-theory of an equicharacteristic discrete valuation ring injects into the $K$-theory of its field of quotients.... .

Mitchell Herbert Taibleson, The failure of even conjugate characterizations of $H^{1}$

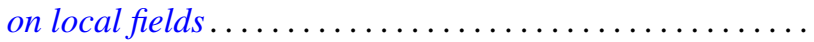

Keti Tenenblat, On characteristic hypersurfaces of submanifolds in Euclidean space ...................................

Jeffrey L. Tollefson, Involutions of Seifert fiber spaces..........

Joel Larry Weiner, An inequality involving the length, curvature, and torsions of a curve in Euclidean $n$-space .......................

Neyamat Zaheer, On generalized polars of the product of abstract homogeneous polynomials.... 